\title{
Virus neutralization of SARS-CoV-2 Omicron variant BA.2 in those vaccinated with three doses of BNT162b2 or CoronaVac vaccines.
}

\section{Malik Peiris ( $\square$ malik@hku.hk)}

University of Hong Kong https://orcid.org/0000-0001-8217-5995

\section{Samuel Cheng}

University of Hong Kong

\section{Karl Chan}

University of Hong Kong

Leo Luk

University of Hong Kong

John Li

University of Hong Kong

Leo Tsang

University of Hong Kong

\section{Leo Poon}

University of Hong Kong https://orcid.org/0000-0002-9101-7953

Chris Ka Pun Mok

Chinese University of Hong Kong https://orcid.org/0000-0002-0525-6772

\section{Susanna SS Ng}

Chinese University of Hong Kong

\section{Fanny Ko}

Chinese University of Hong Kong https://orcid.org/0000-0001-8454-0087

\section{Chunke Chen}

Chinese University of Hong Kong

\section{Karen Yiu}

Chinese University of Hong Kong

\section{Ken Chan}

Chinese University of Hong Kong

\section{David Hui}

The Chinese University of Hong Kong https:// orcid.org/0000-0003-4382-2445

\section{Bosco Lam}

North Lantau Hospital 


\section{Brief Communication}

Keywords: SARS-CoV-2, Omicron, subvariant BA.2, neutralization, vaccine, BNT162b2, CoronaVac

Posted Date: February 8th, 2022

DOI: https://doi.org/10.21203/rs.3.rs-1331606/v1

License: (c) (i) This work is licensed under a Creative Commons Attribution 4.0 International License. Read Full License 


\section{Virus neutralization of SARS-CoV-2 Omicron variant BA.2 in those vaccinated with three doses of BNT162b2 or CoronaVac vaccines.}

Samuel MS Cheng ${ }^{1,8}$, Chris Ka Pun Mok ${ }^{2,3,8}$, Karl CK Chan ${ }^{1}$, Susanna S Ng${ }^{4}$, Bosco HS Lam ${ }^{5}$, Leo LH Luk ${ }^{1}$, Fanny W Ko ${ }^{4}$, Chunke Chen ${ }^{2,3}$, Karen Yiu ${ }^{4}$, John $\mathrm{KC} \mathrm{Li}^{1}$, Ken KP Chan ${ }^{4}$, Leo CH Tsang $^{1}$, Leo LM Poon ${ }^{1,6}$, David SC Hui ${ }^{4,7}$, Malik Peiris ${ }^{1,6}$.

${ }^{1}$ School of Public Health, LKS Faculty of Medicine, The University of Hong Kong, Hong Kong SAR, PR China.

${ }^{2}$ The Jockey Club School of Public Health and Primary Care, The Chinese University of Hong Kong, Hong Kong SAR, PR China.

${ }^{3}$ Li Ka Shing Institute of Health Sciences, Faculty of Medicine, The Chinese University of Hong Kong, Hong Kong SAR, PR China.

${ }^{4}$ Department of Medicine \& Therapeutics, Faculty of Medicine, The Chinese University of Hong, Kong, Hong Kong SAR, PR China.

${ }^{5}$ Department of Pathology, North Lantau Hospital, Hong Kong SAR, PR China.

${ }^{6}$ Centre for Immunology and Infection, Hong Kong Science Park, Shatin, Hong Kong SAR, PR China.

${ }^{7}$ Stanley Ho Centre for Emerging Infectious Diseases, Faculty of Medicine, The Chinese University of Hong, Kong, Hong Kong SAR, PR China.

${ }^{8}$ These authors contributed equally to the research.

\section{Corresponding authors:}

Professor David SC Hui, Department of Medicine and Therapeutics, The Chinese University of Hong Kong, Hong Kong SAR, PR China. E mail: dschui@cuhk.edu.hk

Professor Malik Peiris, School of Public Health, No 7 Sassoon Rd, Pokfulam, The University of Hong Kong, Hong Kong SAR, PR China. E mail: malik@hku.hk 
Key words: SARS-CoV-2, Omicron, subvariant BA.2, neutralization, vaccine, BNT162b2, CoronaVac.

Abstract: SARS-CoV-2 Omicron subvariant BA.2 is increasing in some areas of the world and it is important to assess how well current vaccines may protect against this infection. BioNTech Pfizer (BNT162b2) and CoronaVac are widely used COVID-19 vaccines globally. We determined the $50 \%$ plaque reduction neutralization test $\left(\mathrm{PRNT}_{50}\right)$ and $\mathrm{PRNT}_{90}$ antibody titres to BA.2 virus in sera (twenty each collected 3-5 weeks after third dose) from cohorts vaccinated with three doses of BNT162b2, three doses of CoronaVac, two doses of CoronaVac followed by a third dose of BNT162b2 and those convalescent from SARS-CoV-2 (ancestral virus) (143-196 days after infection). We compared the PRNT titres to BA.2 with titres to BA.1 and ancestral virus. We demonstrate that PRNT 50 and PRNT 90 antibody titres to BA.2 are markedly reduced compared with those to ancestral virus and reduced as much as was observed for BA.1 virus. Those vaccinated with three doses of BNT162b2 or vaccinated with two doses of CoronaVac and a third dose of BNT162b2 develop PRNT antibody titres above the protective threshold from symptomatic infection. Those vaccinated with three doses of CoronaVac fail to achieve protective levels of PRNT 50 antibody to BA.2 subvariant of Omicron 3-5 weeks after vaccination. 


\section{TEXT:}

A new variant of SARS-CoV-2 within Pango lineage B.1.1.529 was recognized in Southern Africa in November 2021 and was classified as "variant of concern" Omicron soon thereafter ${ }^{1,2}$. Three subvariants of Omicron were noted at the time of its first recognition, designated BA.1, BA.2 and BA.3, but the subvariant that initially spread world-wide was BA. $1^{3}$. It had had 37 amino acid changes in the virus spike protein compared to the wild type virus, had increased transmissibility relative to all previous virus variants and rapidly became the dominant SARS-CoV-2 world-wide ${ }^{4}$. It appears to have altered tropism for the human respiratory tract with less efficient replication in the lung parenchyma associated with reduced disease severity ${ }^{5,6}$. A RNA vaccine BioNTech-Pfizer (BNT162b2) and an inactivated whole virus vaccine CoronaVac are two of the most widely used COVID-19 vaccines globally, each with over 2 billion doses delivered so far ${ }^{7}$. We and others have shown that Omicron BA.1 is poorly neutralized by sera from individuals vaccinated with two doses of the $\mathrm{BNT162b2}$ or CoronaVac ${ }^{8,9}$. A third dose of BNT162b2 vaccine was shown to increase neutralizing antibody titres in those previously vaccinated with two doses of BNT162b2 as well as in those vaccinated with CoronaVac. Vaccine effectiveness studies have also shown marked reduction of protection from two doses of RNA vaccines but improved protection associated with a third vaccine dose ${ }^{10}$. The reduction in vaccine protection has prompted the development of Omicron BA.1 specific vaccine ${ }^{11}$.

More recently, Omicron subvariant BA.2 is increasing in a number of countries, apparently out-competing the BA.1 subvariant. BA.2 appears to have even higher transmissibility compared with BA. $1^{12}$. It is of public health importance to assess how well existing vaccine regimens may protect against BA.2. Neutralizing antibody is the best available corelate of protection ${ }^{13}$. Therefore, assessing how vaccine-immune sera neutralize BA.2 will provide assessment of likely protection of existing vaccines against BA.2.

We isolated a BA.2 strain from a COVID-19 patient in Hong Kong in Vero-E6 cells overexpressing TEMPRSS $2^{14}$. We randomly selected 20 sera each, from four previously described cohorts who were convalescent from ancestral SARS-CoV-2 infection or vaccinated with three doses of BNT162b2, CoronaVac or two doses of CoronaVac and third dose of BNT162b2 ${ }^{8}$. The sera were collected at day 143-196 after infection in the SARS-CoV-2 convalescent cohort and 3-5 weeks after the last vaccine dose in the three vaccine-cohorts 
studied. These sera were tested to determine $50 \%$ plaque reduction neutralization test $\left(\mathrm{PRNT}_{50}\right)$ and $\mathrm{PRNT}_{90}$ titres as previously described ${ }^{8}$ (see methods) and data compared with previously reported data on those sera for the ancestral wild-type (WT) or Omicron BA.1 viruses ${ }^{8}$.

BA.2 had markedly reduced PRNT 50 antibody titers relative to WT virus but BA.1 and BA.2 viruses had $\mathrm{PRNT}_{50}$ titers within 4-fold of each other (Figure $1 \mathrm{~A}, \mathrm{~B}$ ). A similar pattern was observed with the more stringent PRNT 90 antibody titres (Extended Data Figure $1 \mathrm{~A}$ ). At 3-5 weeks post the third-dose of vaccine, we found that geometric mean PRNT 50 titer (GMT) (95\% confidence interval) for BA.2 in those given three doses of BNT162b2 was 95.1 (73.7122.8), three doses of CoronaVaC was 9.3 (7.6-11.5), those given two doses of CoronaVac followed by a third dose of BNT162b2 was 46.0 (29.2-72.3) and those convalescent (143-196 days after onset) from SARS-CoV-2 infection was 9.0 (95\% Cl 6.7-12.2) (Figure 1)(Extended data figure 1B). In comparison, GMTs to BA.1 (95\% confidence intervals) for these four groups was 67.3 (45.5-99.6), 8.1 (6.4-10.3), 51.0 (32.9-79.0) and 8.2 (5.5-12.1), respectively. Similarly, GMTs to WT virus (95\% confidence intervals) was 320 (320-320), 65.0 (43.8-96.5), 309.1 (287.5-332.4) and 77.3 (54.1-110.4), respectively.

Khoury and colleagues previously used data from multiple vaccine efficacy trials to establish that a neutralizing antibody threshold associated with $50 \%$ protection from infection was $20.2 \%(95 \% \mathrm{Cl} 14.4-28.4 \%)$ of the geometric mean of titers in convalescent individuals ${ }^{13}$. Using our panel of SARS-CoV-2 convalescent sera collected 30-60 days post infection, we had previously estimated this threshold in our PRNT 50 assay to be a titer of 1:25.6 $(95 \% \mathrm{Cl}$ 1:18.3-1:36.0 $)^{15}$. Applying this $50 \%$ protective threshold to our current $\mathrm{PRNT}_{50}$ titres with BA.2, we estimate that all 20 individuals with three doses BNT162b2, none of 20 with three doses of CoronaVac and 16 (80\%) of 20 vaccinated with two doses of CoronaVac followed by a third dose of BNT162b2 would have protective neutralizing antibody levels 3-5 weeks after the third dose of vaccine (Extended data figure 1B). Preliminary vaccine effectiveness against BA. 2 in the field appears to show increased protection after the third dose of an RNA vaccine $^{12}$. Only one (5\%) of 20 SARS-CoV-2 infection convalescent individuals had protective levels of BA.2 neutralizing antibody. A 4.7-6.4 fold waning of neutralizing antibody has been reported after two doses of BNT162b2 or CoronaVac vaccination ${ }^{16,17}$ and is likely to 
result in reduction in protection even after a third dose of vaccine, as is now being reported in preliminary vaccine effectiveness for Omicron subvariant BA.1 ${ }^{10}$.

Our previous studies and those of others have shown that two doses of BNT162b2 or CoronaVac vaccines did not elicit acceptable levels of neutralizing antibody against Omicron subvariant BA.1. Our current findings suggest that two doses of these vaccines would also not provide protective neutralizing antibody to BA.2 subvariant.

While our data suggest that both BA.1 and BA.2 differ markedly in neutralizing antibody epitopes from the ancestral virus which is the basis for current vaccines, they do not provide information or the antigenic relatedness between BA.1 and BA.2. This requires a reciprocal comparison of BA.1 and BA.2 PRNT titres in sera of individuals convalescent from BA.1 and BA. 2 respectively. The spike proteins of our BA.1 and BA.2 virus isolates have the mutations previously reported by Desingu and colleagues ${ }^{3}$ with BA.1 and BA.2, respectively, having 26 or 24 amino acid substitutions in the spike S1 domain compared to the ancestral virus. Many key antigenically relevant mutations in the spike $\mathrm{N}$ terminal domain (NTD) (e.g. G142D) and receptor binding domain (RBD) (e.g. T478K and E484A) are shared by both variants ${ }^{18}$, which explains why both of them are antigenically distinct from the ancestral SARS-CoV-2. However, each of these subvariants have unique mutations. For example, the NTD of BA.2 does not carry the deletions (HV69del, VYY143del and NL211I) and insertion (215EPE) found in BA.1, but it has a unique deletion (LPPA24S) in its NTD. In addition, the S1 region of BA.1 differs from BA.2 by another 13 amino acid residues (BA.1/BA.2: 19 T/L, $67 \underline{\mathrm{V}} / \mathrm{A}, 95 \mathrm{I} / \mathrm{T}, 213$ V/G, $371 \underline{L} / \mathrm{F}, 376 \mathrm{~T} / \underline{\mathrm{A}}, 405 \mathrm{D} / \underline{\mathrm{N}}, 408 \mathrm{R} / \underline{\mathrm{S}}, 446 \underline{\mathrm{S}} / \mathrm{G}, 496 \underline{\mathrm{S}} / \mathrm{G}, 547 \underline{\mathrm{K}} / \mathrm{T}, 856 \underline{\mathrm{K}} / \mathrm{N}$ and $981 \mathrm{~F} / \mathrm{L} ;$ amino acid residues that are also different form the ancestral sequence are underlined). Many of these amino acid differences are in the N-terminal domain (NTD: 12-306) and receptor binding domain (RBD: 306-527) of spike. The insertion and deletions found in the NTD of BA.1 are known to change the antigenicity of NTD ${ }^{19}$. The NTD of BA.2 has a mutation at position 213, which can affect antibody binding ${ }^{20}$. The RBDs of BA.1 and BA.2 also differ from each other in several key antigenic sites (e.g. 408 R/S, 446 S/G and 496 S/G) ${ }^{18,19}$. This suggests that BA.1 and BA.2 may differ in antigenicity with one another as well as with the ancestral virus. Because we use human sera vaccinated with spike of, or infected with, ancestral virus, we will not be able to detect antigenic differences between BA.1 and BA.2. 
A further limitation of our study is that we have not investigated vaccine-elicited crossreactive $\mathrm{T}$ cell against BA.2. It is known that $\mathrm{T}$ cell responses can contribute to protection from infection and disease ${ }^{21}$ and that $\mathrm{T}$ cell epitopes are substantially conserved between BA.1 and ancestral virus ${ }^{22}$.

In conclusion, three doses of BNT162b2 or two doses of CoronaVac boosted with a third dose of BNT162 are likely to provide protection against Omicron subvariant BA. 2 as well as BA.1. Recovery from ancestral SARS-CoV-2 is unlikely to provide protection from re-infection with BA.2 subvariant.

\section{Acknowledgements}

This research was supported by grants from the Health and Medical Research Fund Commissioned Research on the Novel Coronavirus Disease (COVID-19), Hong Kong SAR (COVID1903003; COVID190126) (CKPM, DSH and MP), US National Institutes of Health (contract no. U01-Grant Al151810) (MP), National Natural Science Foundation of China (NSFC)/Research Grants Council (RGC) Joint Research Scheme (N_HKU737/18) (CKPM and MP), RGC theme-based research schemes (T11-712/19-N and T11-705/21-N) (LLMP, DSH, MP), Guangdong Province International Scientific and Technological Cooperation Projects (2020A0505100063) (CKPM) and C2i (LLMP, MP) administered by Innovation and Technology Commission of Hong Kong. CKMP is the visiting scientist of Lee Kong Chian School of Medicine, Nanyang Technological University, Singapore.

Author contributions statement: MP, DSCH and CKPM planned, coordinated and obtained funding for the study, MP wrote first draft of manuscript, SMSC, CKPM and LLMP coordinated the laboratory testing, sequence analysis and laboratory data analysis, SSN, FWK, CC, KY, KKPC, BHSL coordinated clinical studies and data management, KCKC, LLHL, JKCL, LCKT carried out all biosafety level 3 work for the PRNT testing. All authors commented on the manuscript draft and agreed with its submission.

Competing interests: None of the authors had competing financial or non-financial interests. 
Figure 1 Legend: A. 50\% plaque reduction neutralization test (PRNT 50$)$ titers to ancestral wild-type (WT) virus, Omicron subvariant BA.1 and subvariant BA.2 in those given three doses of BNT162b2 or CoronaVac, two doses of CoronaVac followed by BNT162 and those convalescent from ancestral COVID-19. PRNT 50 data for BA. 2 have been compared with PRNT $_{50}$ data for WT and BA.1 from a previously published cohort (8). Geometric mean titers (GMT) for each group are indicated. Dotted line indicates PRNT 50 threshold of protection and shading indicates $95 \%$ confidence intervals (see text). Mann-Whitney test (two tailed) was used for significance testing. P values are denoted. B. Correlation of BA.2 with BA.1 PRNT $_{50}$ antibody titres. Sera from those with three doses of BNT162b2 (blue dot), three doses of CoronaVac (red square), two doses of CoronaVac and third dose of BNT162b2 (magenta triable) and convalescent from SARS-CoV-2 infection (purple triangle) are indicated. Spearman correlation coefficient with confidence intervals and statistical significance are indicated.

\section{References:}

1. Viana R, et al. Nature doi: 10.1038/s41586-022-04411-y (2022).

2. World Health Organization. 2021. https://www.who.int/news/item/26-11-2021-classification-ofomicron-(b.1.1.529)-sars-cov-2-variant-of-concern

3. Desingu PA, et al. J. Med. Virol. doi: 10.1002/jmv.27601 (2022).

4. Plesner Lyngse F, et al. medRxiv doi: https://doi.org/10.1101/2021.12.27.21268278 (2022).

5. Wolter N, et al. Lancet 399, 437-446 (2022).

6. Hui K.P.Y., et al. Nature doi: 10.1038/s41586-022-04479-6 (2022).

7. Mallapaty S, et al. Nature 600, 580-583 (2021).

8. Cheng S.M.S., et al., Nat. Med. doi: 10.1038/s41591-022-01704-7 (2022).

9. Pérez-Then E., et al. Nat. Med. doi: 10.1038/s41591-022-01705-6 (2022).

10. UK Health Security Agency. Technical briefing 34, January 14th (2022). https://assets.publishing.service.gov.uk/government/uploads/system/uploads/attachment data /file/1050236/technical-briefing-34-14-january-2022.pdf 
11. Waltz E. Nature doi: 10.1038/d41586-022-00199-z (2022).

12. UK Health Security Agency. SARS-CoV-2 variants of concern and variants under investigation in England. Technical briefing 35. 28 January (2022). https://assets.publishing.service.gov.uk/government/uploads/system/uploads/attachm ent_data/file/1050999/Technical-Briefing-35-28January2022.pdf

13. Khoury D.S., et al. Nat. Med. 27,1205-1211 (2021).

14. Matsuyama S., et al. Proc. Natl. Acad. Sci. U S A 117, 7001-7003 (2020).

15. Lau E.H., et al. EClinicalMedicine 41, 101174 (2021).

16. Levin E.G., et al. N. Engl. J. Med. 385, e84 (2021).

17. Zeng G., et al. Lancet Infect. Dis. 7, S1473-3099(21)00681-2 (2021).

18. McCallum M., et al. Science doi: 10.1126/science.abn8652 (2022).

19. Harvey W.T., et al. Nat. Rev. Microbiol. 19, 409-424 (2021).

20. Liu Y., et al. Cell 184, 3452-3466.e18 (2021).

21. Swadling L., et al. Nature 601, 110-117 (2022).

22. Gao Y., et al. Nat. Med. doi: 10.1038/s41591-022-01700-x (2022). 


\section{Methods:}

Clinical specimens: We randomly selected 20 sera from each of four cohorts used for a recently published comparative study of Omicron subvariant BA.1 neutralizing antibody responses 3-5 weeks after vaccination or 143-196 days after SARS-CoV-2 infection ${ }^{(8)}$. The vaccinated cohorts included individuals vaccinated with three doses of BNT162b2, three doses of CoronaVac or two doses of CoronaVac followed by a third dose of BNT162b2. None of these individuals in the vaccine groups had previous history of SARS-CoV-2 infection and this was confirmed with negative IgG to SARS-CoV-2 receptor binding domain antibody in the pre-first vaccine-dose serum. These cohort studies were carried out in Hong Kong during the period $21^{\text {st }}$ February 2020 to November $20^{\text {th }} 2021$ and were approved by the Joint Chinese University of Hong Kong-New Territories East Cluster Clinical Research Ethics Committee (Ref no: 2020.229). All participants provided informed written consent.

Virology: Vero E6 cells overexpressing TMPRSS2 (kindly provided by Dr S Matsuyama and colleagues) ${ }^{(14)}$ were used for virus isolation and plaque reduction neutralization tests (PRNT). Cells were maintained in Dulbecco's Modified Eagle Medium (DMEM) medium (ThermoFisher Scientific), Waltham, MA, USA) supplemented with $10 \%$ fetal bovine serum (FBS) (ThermoFisher Scientific), Waltham, MA, USA) and $100 \mathrm{U} / \mathrm{ml}$ of penicillin-streptomycin (ThermoFisher Scientific), Waltham, MA, USA). The cells used in the study were regularly tested for mycoplasma contamination at the Core Facility, Centre for PanorOmic Sciences, The University of Hong Kong.

The Omicon subvariant BA.2 virus (hCoV-19/Hong Kong/VM22000135_HKUVOC0588P2/2022) isolated in Hong Kong on $3^{\text {rd }}$ January 2022 was used for this study (GISAID EPI_ISL_9570707). The PRNT 50 and PRNT90 data with the BA.2 virus derived from this study was compared with $\mathrm{PRNT}_{50}$ and $\mathrm{PRNT}_{90}$ data obtained for Omicron subvariant BA.1 and ancestral virus obtained for the respective sera previously reported ${ }^{(8)}$.

Plaque reduction neutralization tests were carried out in duplicate using 24-well tissue culture plates (TPP Techno Plastic Products AG, Trasadingen, Switzerland) in a biosafety level 3 facility using Vero E6 TMRESS cells described previously ${ }^{(8,15)}$. Serial two-fold dilutions from 1:10 to 1:320 of each serum sample was incubated with 30-40 plaque-forming units of 
virus for $1 \mathrm{~h}$ at $37^{\circ} \mathrm{C}$. The virus-serum mixtures were added onto pre-formed cell monolayers and incubated for $1 \mathrm{~h}$ at $37^{\circ} \mathrm{C}$ in $5 \% \mathrm{CO}_{2}$ incubator. The cell monolayer was then overlaid with $1 \%$ agarose in cell culture medium and incubated for 3 days, at which time the plates were fixed and stained. Antibody titers were defined as the highest serum dilution that resulted in $\geq 90 \%$ (PRNT 90 ) or $>50 \%$ ( PRNT $_{50}$ ) reduction in the number of virus plaques. This method has been extensively validated on SARS-CoV-2 infected and control sera previously ${ }^{(15)}$.

A virus back titration and a positive control serum was included in every experiment. Virus titres are designated as reciprocal of the serum dilution throughout the text.

\section{$\underline{\text { Statistical analysis }}$}

Sample size calculations: The maximum standard deviation (SD) of log titers for the uninfected vaccinated groups were 1.37 and 1.77 for PRNT50 and PRNT90 respectively. Assuming a 3-fold difference in GMT, a sample size of 10 in each group would have statistical power of $>0.99$ and 0.94 for PRNT50 and PRNT90 respectively, for detecting a difference between groups using two tailed Mann-Whatney $U$ test. Comparisons between groups with larger sample size or smaller within-group variation would have larger statistical power.

Continuous variables were summarized as geometric mean with $95 \%$ confidence interval. Sera with undetectable $(<10)$ antibody titres were assigned an antibody titre of 5 , for purposes of geometric mean titre calculations or statistical comparisons. Comparison between antibody titres to wild type and Omicron variant viruses was done using the two tailed Mann-Whitney $\mathrm{U}$ test. Absolute $\mathrm{P}$ values were provided. $\mathrm{P}$ values $<0.05$ were considered statistically significant.

\section{References:}

8. Cheng S.M.S., et al., Nat. Med. doi: 10.1038/s41591-022-01704-7 (2022).

14. Matsuyama S, et al. Proc. Natl. Acad. Sci. U S A. 117, 7001-7003 (2020).

15. Lau E.H., et al. EClinicalMedicine 41, 101174 (2021). 


\section{Data availability}

Source data (individual anonymized patient and linked laboratory data) and raw antibody data are provided with this paper. The viruses used can be obtained on request to Malik Peiris, E mail: malik@hku.hk 


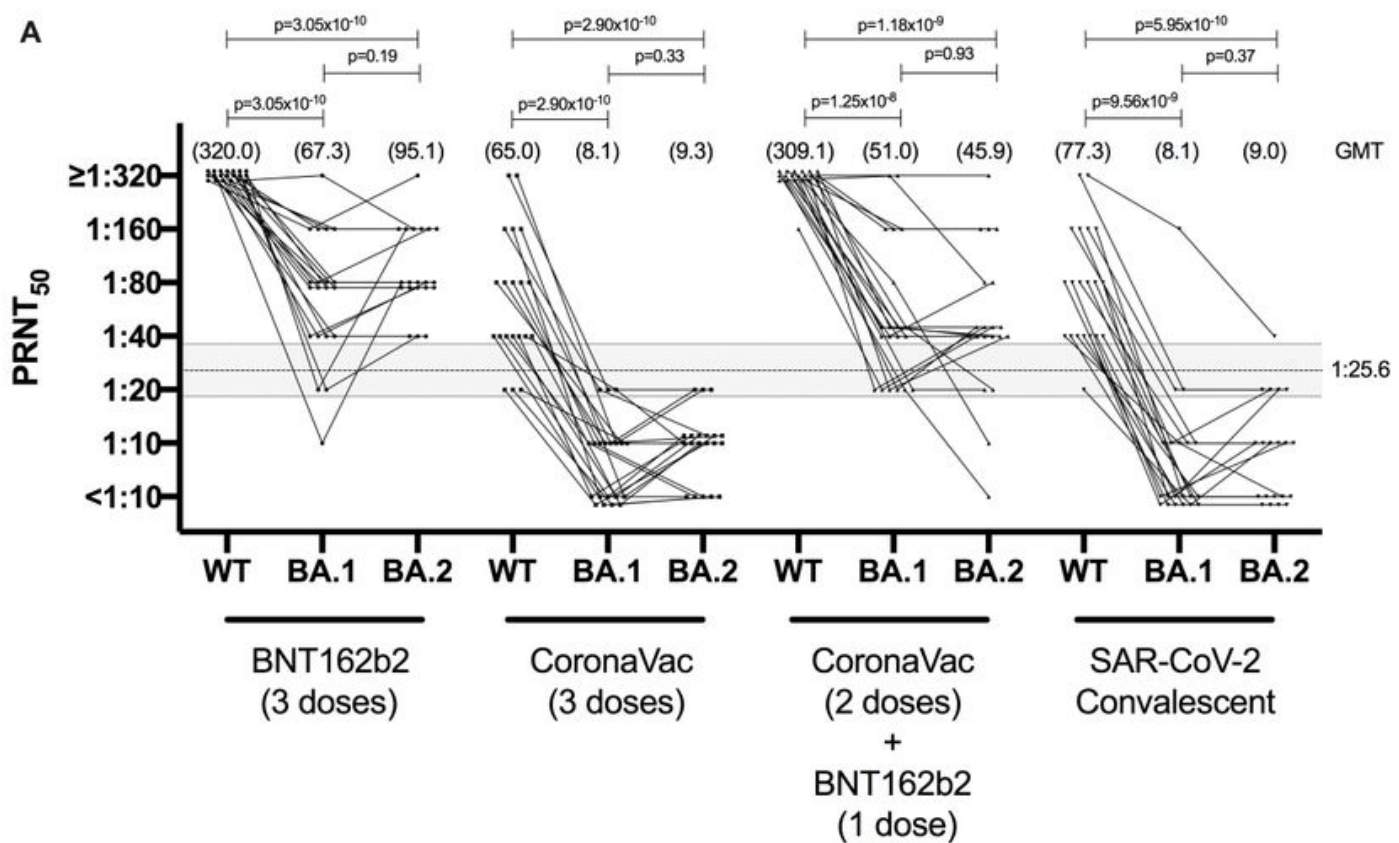

B

BA. 1 vs BA. $2(n=80)$

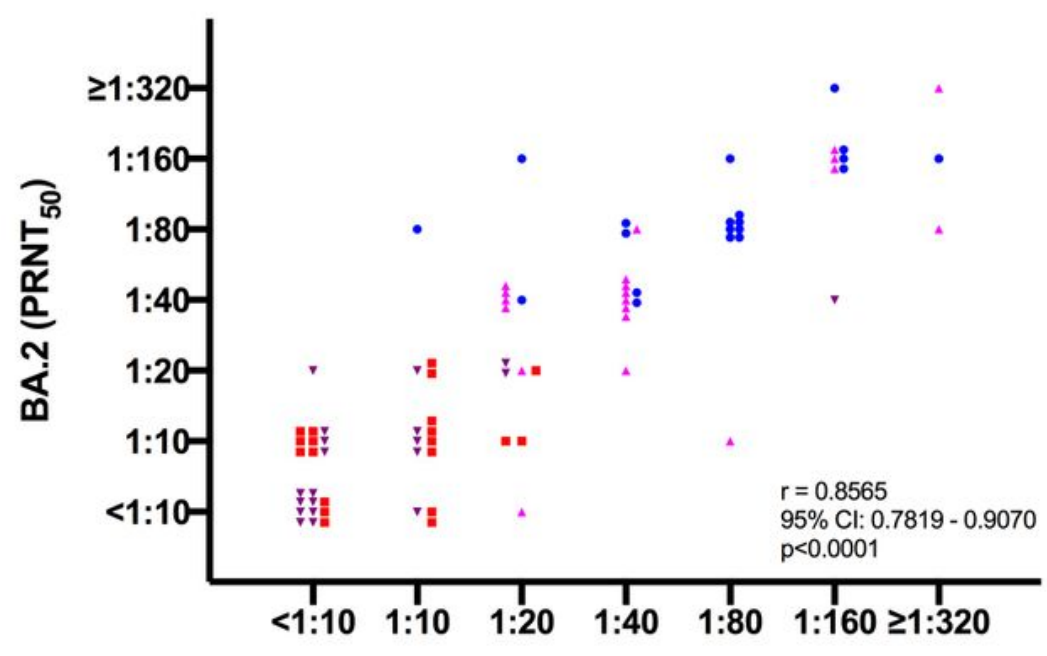

BA.1 (PRNT ${ }_{50}$ )

- BN162b2 (3 doses) - CoronaVac (3 doses)

- CoronaVac $(2$ doses $)+$, SARS-CoV-2Convalescent BNT162b2 (1 dose)

\section{Figure 1}

A. $50 \%$ plaque reduction neutralization test (PRNT50) titers to ancestral wild-type (WT) virus, Omicron subvariant BA. 1 and subvariant BA. 2 in those given three doses of BNT162b2 or CoronaVac, two doses of CoronaVac followed by BNT162 and those convalescent from ancestral COVID-19. PRNT50 data for 
BA.2 have been compared with PRNT50 data for WT and BA.1 from a previously published cohort (8). Geometric mean titers (GMT) for each group are indicated. Dotted line indicates PRNT50 threshold of protection and shading indicates $95 \%$ confidence intervals (see text). Mann-Whitney test (two tailed) was used for significance testing. P values are denoted. B. Correlation of BA.2 with BA.1 PRNT50 antibody titres. Sera from those with three doses of BNT162b2 (blue dot), three doses of CoronaVac (red square), two doses of CoronaVac and third dose of BNT162b2 (magenta triable) and convalescent from SARSCoV-2 infection (purple triangle) are indicated. Spearman correlation coefficient with confidence intervals and statistical significance are indicated.

\section{Supplementary Files}

This is a list of supplementary files associated with this preprint. Click to download.

- Dataset.xIsx 